\title{
Transcription Factor Engineering Harnesses Metabolic Networks to meeting industrial requirements in Yeast
}

\author{
Shuxin Dong ${ }^{1}$, Lei Qin ${ }^{1}$, Chun $\mathrm{Li}^{2}$, and ${\mathrm{Jun} \mathrm{Li}^{3}}^{3}$ \\ ${ }^{1}$ Affiliation not available \\ ${ }^{2}$ Tsinghua University \\ ${ }^{3}$ Beijing Institute of Technology
}

October 31, 2020

\begin{abstract}
Yeast has been well-used as a typical microbial platform to make fermented fine chemicals. However, various stress conditions severely restrict the production costs and benefits. One effective way to resolve such bottlenecks is to engineer transcription factors (TFs) to enhance strain tolerance and production efficiency through remodeling the transcript levels of different stress resistant genes. Here, we focus on the recent advances in the mechanisms of yeast adaptive responses upon stresses of heat, acetic acid and oxidants and classify them into different modules within yeast cells. In particular, novel strategies for the enhancement of both tolerance and yield by TFs engineering are examined. In addition, the applications of artificial transcription factor (ATFs)-based fabricating in metabolic fluxes optimization and quantitative evaluation are discussed. Lastly, we discuss challenges and potential solutions in exploiting TFs engineering and for A bio-based economy products.
\end{abstract}

\section{Introduction}

Yeast is a valuable microbial cell factory in biosynthesis of industrial products, such as biofuels, organic acids terpenoids, abscisic acid and flavonoids[1-5]. However, cell growth rate and product yield are limited in industrial fermentation processes, due to the issues such as heat shock, product toxicities and oxidative stress[6]. Some key reasons are these stresses can cause reactive oxygen species (ROS) accumulation, protein denaturation, chromosome damage, cell membrane destruction and dysmetabolism $[1,7,8]$. Despite the intensive use of physical and chemical ways to reduce the undermines, there are still significant challenges from fermentation process, including the relatively high production cost of extra operations and environment pollution. TFs are important components of complex signaling networks and they can interact with kinases, other transcription factors and metabolite molecules to transmit signals to the nucleus under stress stimulation. So, TFs have the potential to control gene transcription in a specific temporal and spatial scales to improve the complex phenotype $[9,10]$. Recent studies indicate that transcription factor (TFs) have focused on regulating transcriptional level of tolerance genes that contribute to contribute to stress tolerance traits. Therefore, improving the strains tolerance by stress-responded TFs engineering will markedly deal with stress on metabolic networks [11].

Simultaneously, an enhanced understanding of TFs engineering in combination with synthetic biology theory will improve the applications of native TFs and enable the further development of synthetic biology tools. Native stress responsive TFs operated by genetic engineering, helps promote the metabolism of the chassis toward the direction of improving strains tolerance and industrial production. With the new strategies of using TFs springing up, artificial transcription factors (ATFs) which fuse a series of native TFs functional domains have extensive functions. They can make up for the shortcomings of natural TFs and broaden the application fields of TFs engineering. In this review, we briefly review the TF-related molecular traits of 
cellular stress responses and TFs engineering based on TFs regular pattern in improving the stress tolerance and efficiency in biomanufacturing. We also summarize the most recent applications of Artificial transcription factors (ATFs) originated from native TFs, which can either be better used as parts to fine tuning gene expression or for switch to control genes in metabolic pathways than before. Even, it has been invented as devices to quantitatively evaluate metabolites content. Lastly, we discuss the understanding in achieving strains biomanufacturing and innovations of synthetic biology tools by TFs.

\section{Native TFs regulation in inhibitory effect of stress in Yeast}

In order to survive in a dynamic changing environment, yeast has evolved a relatively perfect defense machineries to counteract various environmental stresses under the force of natural selection. These natural mechanisms confer the engineered strains ability on maintain homeostasis in industrial productions process. Highly diverse stresses are transmitted through different response modules to ultimate effectors. Among them, the three most common types of high temperature, acetic acid, and oxidative stress wake up different types of TFs which are part of stress-sensing mechanism system and regulate metabolic networks in yeast.

\section{1}

\section{Heat stress response module}

Heat stress can cause growth and metabolic disturbances during fermentation process, including protein misfolding, electron spillover from the electronic respiratory chain, mitochondrial dysfunction and DNA damage [11-13]. To avoid inhibitions of growth and shutdown of cell metabolism inside microbial cells from stressors, HSF1 , MSN2/4 encoding TFs are the most common regulators in heat stress reaction (HSR), which induced the expression of stress response proteins and molecular chaperones [14-17] (Fig. 1).

Hsf1p is a TF known to be inhibited due to binding with Hsp70 under natural state and it can be phosphorylated and then translocated into the nucleus during heat stress, where they can rapidly recruit transcriptionfriendly mediators (such as Med2p, Med3p, Med15p) and RNA polymerase II to facilitate the transcription of the heat shock protein (HSPs) genes. HSPs genes can maintain the correct structure of proteins and promote the repairing or degradation of damaged proteins [18]. Furthermore, phosphorylation is a booster to Hsflp activity. Under high temperature, the higher the phosphorylation level of Hsf1p is, the better the recruitment of the mediation complex will be. When Hsf1 is combined with Hsp70, its phosphorylation can also promote the transcription level of downstream stress-related genes [19]. In addition, Hsf1p also confers certain degree of resistance to toxic ribosomes through participating in the ribosomal pathway. Two independent TFs, Hsf1p and Ifh1pare involve in the ribosome assembly stress response (RASTR). They are induced by excessive production of orphan r-proteins and then enter into the nucleus to regulate the expression of ribosome synthetic genes and ribosome assembly genes (Fig. 1). In combination with correct operation of ribosome assembly, RASTR has led the generation of cells resistance to irreversible damage caused by incorrect ribosome assembly [20, 21].

In recent years, advanced characterization techniques such as Chromatin immunoprecipitation and deep sequencing (ChIP-seq), mRNA deep sequencing (RNA-seq) and proteomics technology have confirmed the function of another TFs Msn2/4p in high temperature. Msn2/4p mainly regulates yeast stress response via Ras-PKA signaling pathway. Under normal temperature, Ras protein is active, which results high cAMP level and promotes the interaction of cAMP and protein kinase A (PKA) to phosphorylate Msn2/4p, thus Msn2/4p activity are inhibition. Upon heat stress, this inhibition is removed, and, as a result, Msn2/4p can enter the nucleus and bind to the stress response elements (STREs) containing a CCCCT or AGGGG base sequences to activate the transcription of stress response genes [22-24]. Besides, Microarray-assisted, Sequential Window Acquisition of all Theoretical fragment ion spectra (SWATH) and Data independent acquisition (DIA) analyses combined with STRING or SGD database analysis have revealed multiple Msn2/4p-regulated genes and the interacting relationships between different TFs, such as Gcn4p directly interacts with Gal11p, Bas1p, Pho2p, which as a RNA pol II coactivator or several biosynthesis pathways regulator [25-27]. In this high temperature response module, the functions of TFs such as signal cascade transmission, phosphorylation and intracellular substance interactions help us understand the mechanism of high temperature at the 
transcription level and protein folding in yeast.

\subsection{Acetic acid stress response module}

During the bioethanol producing, lignocellulose must go through pretreatment to facilitate downstream reactions $[28,29]$. However, material hydrolysate products, such asacetic acid, furfural and furan undermines strains normal growth and fermentation during pretreatment of biomass [30, 31]. To cope with the effect, the major TF-mediated toxic molecular response systems include transporters, the adaptation of cell membrane components, Hog1p-depend reactions and retrograde regulation contribute to recovery from stress influence (Fig. 2).

Acetic acid is the most abundant byproduct of biomass pretreatment, and when it goes into yeast cell dissociating into two different states of ions, the intracellular $\mathrm{pH}$ drops and the ATP synthesis is disrupted [32]. There are numbers transporters specifically transporting anions and cations on cell membrane to decrease the acetic acid effects in yeast.FPS1 coded aquaglyceroporin channel is generally required for permeating acetate molecules into the cell. In response to acetic acid, active osmoregulation TF Hog1p is phosphorylated and then activates the regulatory factor Rgc2p, which in turn divorces Fps1p and closes aquaglyceroporin channel to prevent acetic acid permeating the yeast cell and to decrease its toxic effects [33, 34]. When the acidity becomes more serious, Hog1p directly interacts with Fps1p and thus causes Fps1p phosphorylated and then degraded by endocytosis [35]. In addition to Fps1p, proton channel protein Pma1p and anion ABC transporters (Pdr10p, Pdr12p and Pdr18p) also assist in resistance to acetic acid [36-38]. Recently, the TFsinvolved retrograde regulation has been discovered as a system to against acetic acid destruction. It is formed by complex comprising subunits (Rtg2p, Rtg1p, Rtg3p and Mks1p) and occurs to alleviate the metabolic disorders caused by the disturbance of mitochondria [39]. Rtg3p-Rtg1p complex activity is induced by the activity of Mks1p-Rtg2p complex, which serves as an activator and be repressed by the Mks1-Bmh1/2p complex. Active Rtg3p-Rtg1p complex smoothly enters the nucleus to stimulate the expression of acetic acid stress-responsive genes, including IDH1/2 $\left(\mathrm{NAD}^{+}\right.$-dependent isocitrate dehydrogenase), DLD3 (D-lactate dehydrogenase) and PYC1 (pyruvate carboxylase). So, retrograde regulation system confers the ability to harness metabolic networks plastic to generate enough glutamic acid for synthesizing other metabolites when mitochondrial activity was impaired [40-46]. Another research reveals that Hog1p as a TF can be induced by overexpressing $O L E 1$ and then sequentially phosphorylate, entering the nucleus to control the expression of a stretch of regulons $(C T T 1, H S P 12$, and STL1) to reconstruct metabolic networks and enhance acetic acid tolerance in yeast [47,48]. In addition, OLE1 encoding the sole $\Delta-9$ desaturase catalyzes saturated fatty acids (SFA) into unsaturated fatty acid (UFA), which is a process known to change of the membrane fluidity in yeast [49]. The cell membranes status such as fluidity and lipid composition can change in order to cope with acetic acid stress. So, the acetic acid module presents the key roles of different types of stress responsive TFs in pathway, including ion channel regulation, retrograde regulation and cell membrane state changes.

\subsection{Oxidative stress response module}

Oxidative Stress is a type of damage in which the original balance of intracellular pro-oxidant/antioxidant is disturbed and metabolic disorders. Sometimes, it due to the accumulation of reactive oxygen species (ROS) which mainly comes from the peroxisomes, endoplasmic reticulum (ER) or mitochondria [50, 51], or it causes by electrophilic compounds, such as cobalt, mercury, furfural and ferulic acid [52-54]. Innately, overcoming oxidative stress depend on different TFs-related signaling which reconstruct metabolic networks in yeast [55]. In addition, some enzymatic or nonenzymatic molecules defense system such as thioredoxin and glutathione also play an important role in ROS detoxification (Fig. 3).

In most ROS-induced regulatory pathways, Yap1p is a major TF that controls the key metabolic networks of oxidative stress resistant systems, including glutathione system, multidrug resistance MDR, glycolysis pathway and cellular detoxification [56]. Upon exposure to inhibitory concentration of ROS, it connecting with scaffold protein Ybp1p forms intramolecular-disulfide bonds between N-terminal and C-terminal the nuclear export signal (NES) is hided, thus inhibit the expression of antioxidant genes. In addition, some 
electrophilic compounds can also direct adduct to C-terminal Cys residue of Yap1, which is activated in a manner independent of Gpx3 [57, 58]. For instance, Hyr1p encoding thiol peroxidase catalytic catalyzes the formation of intramolecular disulfide bonds in Yap1p, which inhibits Yap1p nuclear outputting and accumulating in the nucleus. And, to cope with oxidized proteins by ROS, Ire1-Hac1 pathway of unfolded protein response (UPR) maintains endoplasmic reticulum homeostasis and correct protein folding in yeast. Ire1p is an endonuclease located on endoplasmic reticulum. When senses the unfolded protein, it activates Hac1p by splicing introns. Hac1p as a TF regulating the UPR to cope with ROS, and it transcriptionally induces a number of genes regulating growth and mitochondria homeostasis of yeast [59,60]. Besides, the thioredoxin and glutaredoxin systems involve in maintain redox state in strains. They utilize proteins with reductive amino acid residues to reduce oxidizing molecules. At the same time, various reductases use $\mathrm{NADPH}$ as an electron donor to transform into an oxidized state to achieve recycling. The mainly three oxidative responsive TFs (Yap1p, Ire1p and Hac1p) forms part of the classical oxidative stress response module and occupies an important position in maintaining redox state and reconstruction metabolic pathways in yeast. And some reductive molecules as another branch of the oxidation response system help to eliminate oxidizing molecules to maintain strains homeostasis.

\section{Conventional TFs engineering implicated in meetingindustrial requirements}

A clear correlation between the strains industrial value and TFs engineering reveals that proper reconstruction of gene transcription network can change organelle structure and pathway flux into the direction of enhancing strains tolerance and improving productivity, which has shown to be more important during strain modification. In combination with genetic modification or increasing the diversity of genetic information by heterologous expression and global transcription machinery engineering (gTME), conventional TFs engineering will markedly enhance the generation of cells resistance to various stress and arise with the desired phenotype.

\subsection{G enetic modification of native TFs}

With many omics and genome sequencing datasets providing valuable understanding of tolerance traits, environmental stress responsive native TFs functioning as activators or inhibitors have been repeatedly overexpressed or knocked-out in harnessing metabolic networks in yeast[61, 62]. For example, HAA1 is a TF which not only directly controls stress-induced genes expression such as sphingolipid and ceramide metabolism (YPC1), carbohydrate metabolism (TOS3), and cell wall related secretory glycoprotein (YGP1), but also indirectly regulates Msn4p, Nrg1p, Fkh2p, Stp4p, Com2p and other TFs in response to acid stress [63]. The above regulated genes have played important roles in prevent stress-induced cellular dysfunctions. After overexpressing HAA 1 treatment, modified xylose strains tolerated an initial acidity level of $50 \mathrm{mM}$ acetic acid, $50 \mathrm{~g} / \mathrm{L}$ xylose environment and increased the ethanol yield from $0.26 \mathrm{~g} / \mathrm{g}$ to $0.32 \mathrm{~g} / \mathrm{g}$. In addition, engineered strains could also grow in non-detoxified hardwood hydrolysate which contain glucose, xylose, acetic acid, furfural and other inhibitors, and the utilization rate of glucose and xylose had been improved in yeast [64, 65]. Similarly, overexpressing Whi2p interacted with Prs1p leaded multiple genes upregulated in vivo, such as Catalase (CTA1), glutathione antioxidant system (GSH1), reducing furan aldehyde enzymes $\left(A D H^{7}\right.$ ), global TFs (Msn2/4 ) [66]. Further, asides increasing strains tolerance, TFs engineering can promote the synthesis of natural products such as squalene, glycyrrhizic acid, and glycyrrhetinic acid and changes the xylose conversion rate. For instance, overexpressing INO2 had improved squalene production in engineered yeast. Ino2p and Ino4p are TFs that regulate lipid synthesis, while Opi1p acts as a negative regulator to inhibit the activity of Ino2p and Ino4p. By overexpressing INO2, the area of the endoplasmic reticulum had been expanded to provide a high-quality microenvironment for the enzymes (ERG9, HMG1 and PPDS ) in the MVA pathway, resulting in increasing glycolysis flux and acetyl-CoA content and enhancing protein synthesis capacity squalene production(634 mg/L) [67].

Besides overexpressing is used to conversion of sensitive phenotype to valuable microorganisms, yeast has the potential to convert lignocellulose into ethanol only by introducing an effective xylose metabolizing pathway[68]. Heterologous xylose system can effect native metabolic networks, and the transcription level of some important genes were down-regulation, such as in carbon metabolism (FBA1, GPM1 and TDH2), 
DNA damage stimulus respond and repair (HTA2), structural constituent of ribosome (RPL22A, RPL22B and RPL7A) during the GX stage (glucose-xylose co-fermentation). And, cell cycle-related or vitamin metabolic-related TFs Nrm1p, Yhp1p, and Thi2p had also slightly down-regulation. Thi2p is a thiamine biosynthetic TF that interacts with Thi3p and Pdc2p and regulates the transcription of a number of target genes. Knocking out THI2(encoding Thi2p) resulted in the upregulation of genes involved in ribosome and transcription initiation factor complex subunits synthesis and energy metabolism, which had positive effects on the utilization of xylose in the GX stage (increase 67.7\%). Even though, an efficient xylose utilization in the THI2 knocked-out yeast strain is limited by carbon source conditions, in which glucose responding system (Snf3p/Rgt2p, Snf1p/Mig1p and cAMP/PKA) still strongly controlled the initiation of signaling processes in fermentative metabolism. Therefore, knocking out of THI2 didn't increase the utilization of xylose in the $\mathrm{X}$ stage(xylose fermentation)) [69, 70].

Another study investigating ethanol production displays critical role played by TFs of chromatin remodeling (such as Swi3p ) in knock-out strains [71]. SWI/SNF (BAF) Chromatin Remodeling Complex utilizes the energy released by ATP hydrolysis to drive the movement of nucleosomes, which facilitates chromatin structure remodeling and promotes genes expression of ethanol dehydrogenase (ADH1/2) and galactokinase (GAL1 andSUC2 ). So, reducing ADH2 expression might be one of the major contributions to ethanol production by knocking out SWI3 which encodes a subunit of SWI/SNF chromatin remodeling complex [72-76].

\section{2 Heterologous expression of native TFs}

Introducing heterogeneous stress-related TFs genes realizes the expression of dominant genes from different sources. It breaks through the genetic information communication barrier between different species to meet the purpose of acquiring new genetic material quickly. TFs engineering using heterologous expression had also been proven to effectively modify the phenotypes in plants (Table 1), while this method had been reported in yeast. Transforming the Kluyveromyces marxianus TF Msn2p or Hsf1p inSaccharomyces cerevisiae favorably led the bioethanol production up to $27.6 \mathrm{~g} / \mathrm{L}$ and $27.2 \mathrm{~g} / \mathrm{L}$ respectively in the presence of $104.8 \mathrm{~g} / \mathrm{L}$ glucose, $43^{\circ} \mathrm{C}$, and the best one made a $46 \%$ higher than control. One reason was due to DNA binding domain was different from primitive structure [77, 78]. And, transcriptomic analysis revealed that the reconstruction of lipid metabolism, glucose transport and glycolysis or gluconeogenesis process display critical role played by TFs of heterologous expression in enhancing ethanol tolerance and ethanol production. So, TFs engineering can harness metabolic networks to obtain industrial strains and apply for different organisms.

\subsection{Modified TFs}

gTME based on changing genetic information by error-prone PCR offers a selective advantage to building tolerant mutants. As a proof-of-strategy, researchers created a mutant library of the TF SPT15 encoding TATA-binding protein, followed by a screening to identify mutants showing tolerance improvement. Using this strategy, the authors were able to obtained a mutant (Phe177Ser, Tyr195His, Lys218Arg) that recovered growing normally under $6 \%$ ethanol and $120 \mathrm{~g} / \mathrm{L}$ glucose and improved fermentation capacity. These results illustrated that change in Tyr195His amino acid and the synergistic interaction of the other two amino acids at the mutant site for the impact of inhibited effect of NC2 negative regulators on SPT15 and the interaction with the subunit SPT3-SAGA of the transcriptional regulatory complex, which leading to the genes transcription level and metabolic networks reconstructed [9]. ThePingkai Ouyang team also used mutate TF SPT15 adjust the pathways of glycolysis, respiration, gluconeogenesis and PPP in GX stage [79], and obtained high efficiency strains in the mixed medium of xylose and glucose. The utilization rates of xylose and glucose were up to $90.8 \%$ and $97.3 \%$, respectively [80]. Furthermore, Transcription initiation complexes included TFs were operated by gTME. For instance, transcription factor D (TFIID) and Spt-Ada-Gcn5pAcetyltransferase (SAGA) which jointly possess TATA-box binding protein (TBP) associated factors encoded by SPT3 and SPT8 are involved in the gene transcription process [81-84]. Spt3p and Spt8p as TFs can interact with TBP, and thereby involve nucleosome subunits deacetylated which is necessary for the initialization of transcription process. Although the SAGA complex binds to TBP via Spt3p, it is easier for Spt8p to bind to TFIIA and TBP than Spt3p. Mutated Spt8p changed the force, distance and quantity of hydrogen bonds 
that maintained the protein structure, leading to simultaneously alter its activity and charge transfer system. Finally, the ethanol tolerance and ethanol yield of the strains were increased [85]. Further, researchers used Random Insertional-deletional Strand Exchange (RAISE) technology to build Spt3p mutagenesis library and screened strain which could survive under the environment of $1.8 \mathrm{~g} / \mathrm{L}$ lead. The degree of change in gene expression and the relationship between the tolerance phenotypes and gene genotypes analyses had identified that altered trehalose accumulation by mutated TF have contributed to enhanced yeast tolerance [86]. Interestingly, researchers mutated methylotrophic yeast (O.thermomethanolica) TF HAC1 by CRISPRCas9 technology to improve Endoplasmic reticulum (ER) resistant and desired product yield. Although strains had not been associated with any noticeable phenotypes in the resistant to ER stress, the mutation efficiency was $63 \%$. It may be recommended that CRISPR-Cas9 has better mutation efficiency than gTME and proposed other option for mutant TF for future study [87].

\section{Designed Artificial transcription factors}

With the development of gene editing, high-throughput testing, bioinformatics, computational biology, researchers are no longer limited to engineer native TFs to improve strains tolerance and regulate the biosynthesis of interest products, they have attempted to developed powerful TFs in reconstruct strains metabolism for meet industrial requirements and as a tool to use in genetic biosensor. ATF is one type of powerful TFs originated from native TFs and its important domains, such as DNA-binding domain (DBD) and Effector domain (ED) are rational designed to suit various applications [88]. The type of DBD are from native zinc fingers (ZFs), transcription activator-like effectors (TALEs), catalytically inactive form of Cas9 (dCas9) [89], while ED are divided into activator (VP16, VP64, CRP and Gal4) and repressor (SID, KRAB, EAR, SRDX and Ume6) [90, 91]which can co-interact with transcription-related components in the cell to achieve transcription control [92]. The combination of function domains selection and integration in the ATF construction is imperative to give the transcriptional performance and develop specialized bio-function devices. So, ATFs have certain potentials as transcription remodeling by enhancing transcriptional activity, creating orthogonal systems and adding bistable switch, even has been used to predict genes associated with tolerance by the specificity of the structure binding to DNA. In addition, while the biosensor devices constructed by the conventional TFs system still bring new practical tools for synthetic biology, ATFs also supply the deficiencies of them and expands their application scope.

\subsection{ATFs as transcriptionalreconstruction partconstruct industrial chassis cells}

Constructing chassis cells that can meet production needs is a research direction in synthetic biology. Due to a serious of pathway of growth, metabolism, cell cycle, or endoplasmic reticulum are regulated by TFs, they are commonly used as part for genetic modification to reconstruction of gene transcription network. However, the known TFs sometimes fail to satisfy more elaborate and complex regulation, and even their original effects will be diluted by the complex metabolic network within the cell. Several specific ATFs have been implicated in the reconstruction part to avoid these problems and to improve strains tolerance and production capacity. For example, Cys2-His2 zinc finger domain is common DBD, which generally exists in the bZIP family [93]. And, TFs in this family are usually associated with tolerance. Researchers selected 40 human-derived ZFs and fused them with the CRP domain (interact with RNA polymerase) of E. coli to construct combinatorial libraries of ATFs. After transformed them into E. coli and screening found that the combination of Z13, Z2 and Z25 resulted in the upregulation of genes (ompW, cpxP ,yliH and ybaT ) linked with heat-resistant. Similarly, genes negatively related to heat tolerance $(\operatorname{mar} R, \operatorname{mar} A$ and $\operatorname{mar} B$ ) were significantly down-regulated [94]. Further study also found the combination of truncated CRP and three ZFs (Z11, Z4, and Z23) could enable the strain to survive in the environment of $1.5 \%$ (vol/vol) butanol, and this hybrid TFs were expedient in improving heat and butanol tolerance of E. coli [95]. Hence, this strategy of ATF with hybridized different domains also can favorably enhance yeast tolerance by regulate metabolic networks related genes.Jin-Soo Kim et al . respectively chosed 25 and 40 ZFs to create a pool of multiple ATFs containing random combinations of three or four ZFs coupled to either an activation or inhibition domain. It had been noted that different combinations leaded to the different acquisition of tolerance to heat shock $(52)$ and ketoconazole $(50 \mu \mathrm{M})$ and swapping two different ED showed a reversed phenotype. 
Besides, PDR5 and YLL053C were confirmed to participate in conferring ketoconazole resistance in yeast. Subsequently, the acetic acid-tolerant strains were also obtained by this ATFs library [96, 97]. It was not difficult to noted that ATFs play a positive role in enhancing the stress tolerance and discovering new regulated genes.

\subsection{Gene Expression System withATFs switch}

Despite the intensive use of native elements in switches design, there is still limitation of the quantity and quality, including the rarely response parts and ability of regulation, orthogonality or plasticity. Variable structure and wider range utilization of ATFs, lead to a great interest in development of expression switch with novel properties. The fine modulation of gene expression by ATF-related switch will be of benefit to promote the subsequently multilayered metabolic pathways run accurately such as decoupled the two states of the growth and production. Additionally, universal ATF switch breaks species limitations for metabolic network reconstruction.

A study revealed that two ATFs with opposite effect could establish for a bistable switch. The repressors consisted of DNA binding domain (SrpR, PhlF, TetR, Bm3R1, and LacI) and the SV40 nuclear localization signal (NLS), and the activators had the same NLS, threshold of the more types of DNA binding domain (TarA, Orf2, LexA, and TetR) and additional C-terminal VP16 activation domain. So, paired with the corresponding inducible promoters (GAL1 and THI4 prompter), the bistable switch could thus be well suited even for repressing or activating the expression of regulated genes, and switched between two states. For instance, this ATFs-based bistable switch was used to fine-tune the expression of VioC and VioD which were the parts of heterologous violacein pathway, the metabolism pathway successfully reconstructed and developed towards the synthesis of proviolacein (green pigment), deoxyviolacein (red pigment) and violacein (violet pigment) [98]. Besides, a period motif or the whole of TFs from the Arabidopsis coupled to heterologous AD and their cognate binding sites were constructed an orthorhombic gene expression switch together in yeast. In this system, some ATFs with high output combinations (e.g., NLS-GAL4AD-JUB1and NLS-GAL4AD-ATAF1) which exceeded the strong constitutive yeast TDH3 promoter were identified by detecting the strength of yEGFP, and a link between the copy numbers of DNA-binding sites and plant-derived ATFs transcriptional output were suggested [99]. Employing the mentioned above ATFs library, researches also created COMPASS which was a high-throughput cloning method. And this artificial constructed orthogonal transcription system balanced the multiple genes expression and decoupled the two states of the growth and production, achieving an increase in the target product yield in Saccharomyces cerevisiae[100]. Moreover, dCas9-based or TALEs-based ATFs were also used in orthogonal systems. TALEs is DBD derived fromXanthomonas spp and no endonuclease activity dCas9 is a transcription activator be guided to a $20 \mathrm{bp}$ DNA target by a small single RNA derived from Streptococcus pyogenes. After the two were respectively fused with AD (VP4 and VP64), they bound to the matched synthetic promoter $\left(\mathrm{P}_{\mathrm{Syn}}\right)$ to regulate the genes expression and satisfied a wide range of inducible expression strengths. ATF-related switch was indispensable for the design of orthogonally regulated signaling and metabolic network. And, among various causes of ATF-related switch plasticity, the number and direction of binding sites (BS) were considered as the main influencing factors, which associated with the spectrum of transcriptional outputs owing to the number of transcription initiation complexes recruited by BS. Further, a steric hindrance effect caused by the oriented BSs and spacers also affected the interaction between $\mathrm{AD}$ and the basal transcription machinery or TFs. If people want to fine-tune the ATFs regulation intensity, it can be achieved via introducing 1-2 single nucleotide mismatches into the BSs [101].

Although the above expression system can meet orthogonality, the application is still limited by creating matching promoter which increases the difficulty of large-scale application to engineered strains. Therefore, a flexible and highly adaptable ATFs system is needed to satisfy industrial needs. A suggested that the synthetic expression system (SES) constituted with core promoters (CPs) which from S. cerevisiae, Aspergillus niger and Trichoderma reesei, ATFs (LexA-VP16 or Bm3R1-NLS-VP16) as well as BSs achieved working in different host such as Saccharomyces cerevisiae ; Yarrowia lipolytica ; Aspergillus niger ;Pichia pastoris; Trichoderma reesei and Pichia kudriavzevii . And, four strategies, involving changing the number or type 
of CP, optimizing codons for ATFs act respective to switching intensity [102]. Overall, ATFs have expanded the switch type for modifying metabolic pathways.

\subsection{ATFs-dependent device applicated inquantitatively evaluate}

Upon ATFs development drove by the design concept of synthetic biology, modular synthetic devices were invented. In general, they are used to evaluate metabolites of interest and optimized by adjusting the adaptability and diversity of functional elements.

Sometimes, TF-based biosensors have huge potential in high-throughput screening. For example, the developed 3-hydroxypropionic acid (3-HP) biosensors consisted with LysR-type transcriptional regulator (LTTR), MmsR TF protein, $\mathrm{P}_{\mathrm{mmsA}}$ promoter and GFP reporter gene were involved in the response of the concentration of 3-HP inPseudomonas denitrificans which covered a wide dynamic range (0-100mM) [103]. To take advantage of the TFs sensitivity and avoiding narrow detection ranges of native TFs, ATFs biosensors system was introduced in the strain to monitor and respond metabolites in vivo. Studies have shown that a orthogonal ATF-based cAMP detection device to mammalian cells consisted of cAMP receptor protein CRP from E.coli and VP16 transactivation domain from Herpes simplex virus. And, this tool was constructed into two plasmids, the one contained ATF which combined with intracellular cAMP and another as a reporter part contained a single CRP specific operator site OCRP that could be bind to ATF and a chimeric promoter. Compared with the traditional CRE reporter device, the new detection device avoided the interference of calcium signaling, MAPK signaling or Rho signaling, and achieved accurate monitoring of cAMP signals in mammalian cell populations [104]. Although traditional TF-based biosensors are easy to application in screening small molecules such as Itaconic acid, flavonoids, mevalonate [105], ATFs biosensors lack of creation in Eukaryote such as yeast.

\section{Conclusions}

As a regulator of the expression of genes, TF disturbed at the transcriptional level acted like a domino effect, affecting the whole metabolic network in yeast. With cascade multiple signaling pathways of TFs in stress have been identified from research, conventional TFs engineering can contribute to modified strains in combination with theoretical basis, which may give strains new phenotypes and avoid the limitations of some engineering methods. At the same time, he emergence of ATFs originated from native TF has promoted the development of conventional TFs engineering towards the diversification of functions. ATFs can not only meet the intervention in enhancing transcriptional activity and reconstructing metabolism, but also be designed as standardized components to expand the number of synthetic biological tools, such as ATFs switch or ATFs biosensors, which have been shown to be more effective in controlling gene expression or quantitatively evaluating interest products compared with native TFs. And, the development of ATFsbased biosensors provides prospects for screening valuable engineering strains in the future. TFs engineering harnesses metabolic networks becoming increasingly active various directions in yeast.

There are several challenges underlying the application of TFs engineering. Transcriptomic, proteomics, GC-MS, and QTL (Quantitative trait locus) analysis of yeast response to stress revealed determine changes in metabolic fluxes and multiple genes expression levels. These can find more meaningful key TFs, and then improve strains tolerance by adjusting or combinatorial regulating them. However, sometimes the effect is not very effective $[65,106]$. It due to the intricacies in the regulation of TFs as well as determined by specific circumstances, it has become a difficulty to objectively characterize the strain under the stress. Besides, the functional redundancy of TFs negatively affects the study of gene function. Truly showing the characteristics of binding to target genes and might be necessary for future research [107]. Although there has been a robust engineered strain by traditional stress-resistant system based on understanding of the growth and metabolism mechanism, the chassis for high productivity is still limited by less novel defense means. So, researchers not only need to pay attention to existing regulatory network, but also devoted to taped new TFs or transform known TFs to build a "unique" defense system, which causes a high-quality chassis cell factory achieve efficient production of target products $[108,109]$. Even, actively modifying strains phenotype would be energetically a less costly and convenient if creating a universal ATFs which would not 
restrict by species.

In conclusion, TFs engineering harnessed metabolic networks has power and ability to avoid the limitations of methods including laboratory evolution, random mutation, and many others and achieves reconstruction metabolic networks under stress as advanced genetic tools in yeast. With these continuous efforts, it will soon be a mainstream strategy for improving strains tolerance and the biosynthesis of industrial products in engineered chassis. Similarly, ATF originated from native TFs improved capacity in metabolic networks design promotes the research of TFs engineering advancement.

\section{Acknowledgement}

This work was financially supported by the the Natural Science Foundation of China (21808013)

\section{Conflict of interest}

The authors declare no conflict of interest.

\section{References}

1. Caspeta, L.,Castillo T., Nielsen J., Modifying Yeast Tolerance to Inhibitory Conditions of Ethanol Production Processes. Front Bioeng Biotechnol 2015, 3, 184.

2. Kuroda, K.,Hammer S.K.,Watanabe Y.,Montano Lopez J.et al., Critical Roles of the Pentose Phosphate Pathway and GLN3 in Isobutanol-Specific Tolerance in Yeast. Cell Syst 2019, 9, 534-547 e535.

3. Zhu, Z.,Hu Y.,Teixeira P.G.,Pereira R.et al., Multidimensional engineering of Saccharomyces cerevisiae for efficient synthesis of medium-chain fatty acids. 2020, 3, 64-74.

4. Pereira, R.,Wei Y.,Mohamed E.,Radi M.et al., Adaptive laboratory evolution of tolerance to dicarboxylic acids in Saccharomyces cerevisiae. Metab Eng 2019, 56, 130-141.

5. Otto, M.,Teixeira P.G.,Vizcaino M.I.,David F.et al., Integration of a multi-step heterologous pathway in Saccharomyces cerevisiae for the production of abscisic acid. Microb Cell Fact 2019, 18, 205.

6. Snoek, T.,Verstrepen K.J., Voordeckers K., How do yeast cells become tolerant to high ethanol concentrations? Curr Genet 2016, 62, 475-480.

7. Auesukaree, C., Molecular mechanisms of the yeast adaptive response and tolerance to stresses encountered during ethanol fermentation.J Biosci Bioeng 2017, 124, 133-142.

8. Gibson, B.R.,Lawrence S.J.,Leclaire J.P.,Powell C.D.et al., Yeast responses to stresses associated with industrial brewery handling.FEMS Microbiol Rev 2007, 31, 535-569.

9. Alper, H.,Moxley J.,Nevoigt E.,Fink G.R.et al., Engineering Yeast Transcription Machinery for Improved Ethanol Tolerance and Production.Science 2006, 314, 1565-1568.

10. Chen, Z.,Zheng Z.,Yi C.,Wang F.et al., Intracellular metabolic changes in Saccharomyces cerevisiae and promotion of ethanol tolerance during the bioethanol fermentation process. RSC Advances 2016, 6, 105046-105055.

11. Gao, L.,Liu Y.,Sun H.,Li C.et al., Advances in mechanisms and modifications for rendering yeast thermotolerance. J Biosci Bioeng 2016, 121, 599-606.

12. Xu, K.,Gao L.,Hassan J.U.,Zhao Z.et al., Improving the thermo-tolerance of yeast base on the antioxidant defense system. Chemical Engineering Science 2018, 175, 335-342.

13. Xu, K.,Lee Y.S.,Li J., Li C., Resistance mechanisms and reprogramming of microorganisms for efficient biorefinery under multiple environmental stresses. Synth Syst Biotechnol 2019, 4, 92-98.

14. Kovacs, D.,Sigmond T.,Hotzi B.,Bohar B.et al., HSF1 Base: A Comprehensive Database of HSF1 (Heat Shock Factor 1) Target Genes. Int J Mol Sci 2019, 20, 5815. 
15. Kuang, Z.,Ji H., Boeke J.D., Stress response factors drive regrowth of quiescent cells. Curr Genet 2018, 64, 807-810.

16. Jethmalani, S.M., Henle K.J., Interaction of heat stress glycoprotein GP50 with classical heat-shock proteins. Exp Cell Res 1998, 239, 23-30.

17. Li, S.,Giardina D.M., Siegal M.L., Control of nongenetic heterogeneity in growth rate and stress tolerance of Saccharomyces cerevisiae by cyclic AMP-regulated transcription factors. PLoS Genet 2018, 14, e1007744.

18. Richter, K.,Haslbeck M., Buchner J., The heat shock response: life on the verge of death. Mol Cell 2010, 40, 253-266.

19. Zheng, X.,Krakowiak J.,Patel N.,Beyzavi A.et al., Dynamic control of $H s f 1$ during heat shock by a chaperone switch and phosphorylation. Elife 2016, 5, e18638.

20. Albert, B.,Kos-Braun I.C.,Henras A.K.,Dez C.et al., A ribosome assembly stress response regulates transcription to maintain proteome homeostasis. Elife 2019, 8, e45002.

21. Tye, B.W.,Commins N.,Springer M.,Pincus D.et al., A risk-reward tradeoff of high ribosome production in proliferating cells.bioRxiv 2018, 458810.

22. Chowdhary, S.,Kainth A.S.,Pincus D., Gross D.S., Heat Shock Factor 1 Drives Intergenic Association of Its Target Gene Loci upon Heat Shock. Cell Rep 2019, 26, 18-+.

23. Elfving, N.,Chereji R.V.,Bharatula V.,Bjorklund S.et al., A dynamic interplay of nucleosome and Msn2 binding regulates kinetics of gene activation and repression following stress. Nucleic Acids Res 2014, 42, 5468-5482.

24. Kobayashi, N., McEntee K., Identification of cis and trans components of a novel heat shock stress regulatory pathway inSaccharomyces cerevisiae. Mol Cell Biol 1993, 13, 248-256.

25. Vamvakas, S.S.,Kapolos J.,Farmakis L.,Koskorellou G.et al., Ser625 of msn2 transcription factor is indispensable for ethanol tolerance and alcoholic fermentation process. Biotechnol Prog 2019, 35, e2837.

26. Satomura, A.,Katsuyama Y.,Miura N.,Kuroda K.et al., Acquisition of thermotolerant yeast Saccharomyces cerevisiae by breeding via stepwise adaptation. Biotechnol Prog 2013, 29, 1116-1123.

27. Xiao, W.,Duan X.,Lin Y.,Cao Q.et al., Distinct Proteome Remodeling of Industrial Saccharomyces cerevisiae in Response to Prolonged Thermal Stress or Transient Heat Shock. J Proteome Res 2018, 17, $1812-1825$.

28. Martins, L.H.,Rabelo S.C., da Costa A.C., Effects of the pretreatment method on high solids enzymatic hydrolysis and ethanol fermentation of the cellulosic fraction of sugarcane bagasse.Bioresour Technol 2015, $191,312-321$.

29. Johnson, E., Integrated enzyme production lowers the cost of cellulosic ethanol. Biofuel Bioprod Bior 2016, 10, 164-174.

30. Zhang, M.M.,Xiong L.,Tang Y.J.,Mehmood M.A.et al., Enhanced acetic acid stress tolerance and ethanol production in Saccharomyces cerevisiae by modulating expression of the de novo purine biosynthesis genes. Biotechnol Biofuels 2019, 12, 116.

31. Thompson, O.A.,Hawkins G.M.,Gorsich S.W., Doran-Peterson J., Phenotypic characterization and comparative transcriptomics of evolvedSaccharomyces cerevisiae strains with improved tolerance to lignocellulosic derived inhibitors. Biotechnol Biofuels 2016, 9, 200.

32. Palma, M.,Guerreiro J.F., Sa-Correia I., Adaptive Response and Tolerance to Acetic Acid in Saccharomyces cerevisiae andZygosaccharomyces bailii : A Physiological Genomics Perspective.Front Microbiol 2018, 9, 274. 
33. Lee, J.,Reiter W.,Dohnal I.,Gregori C.et al., MAPK Hog1 closes the S. cerevisiae glycerol channel Fps1 by phosphorylating and displacing its positive regulators. Genes Dev2013, 27, 2590-2601.

34. Lee, J., Levin D.E., Rgc2 Regulator of Glycerol ChannelFps1 Functions as a Homo- and Heterodimer with Rgc1 .Eukaryot Cell 2015, 14, 719-725.

35. Zhang, J.G.,Liu X.Y.,He X.P.,Guo X.N.et al., Improvement of acetic acid tolerance and fermentation performance of Saccharomyces cerevisiae by disruption of the FPS1 aquaglyceroporin gene.Biotechnol Lett 2011, 33, 277-284.

36. Ullah, A.,Orij R.,Brul S., Smits G.J., Quantitative analysis of the modes of growth inhibition by weak organic acids in Saccharomyces cerevisiae. Appl Environ Microbiol 2012, 78, 8377-8387.

37. Piper, P.W., Resistance of yeasts to weak organic acid food preservatives. Adv Appl Microbiol 2011, 77, $97-113$.

38. Teixeira, M.C.,Godinho C.P.,Cabrito T.R.,Mira N.P.et al., Increased expression of the yeast multidrug resistance $\mathrm{ABC}$ transporter $P d r 18$ leads to increased ethanol tolerance and ethanol production in high gravity alcoholic fermentation. Microb Cell Fact 2012, 11, 98.

39. Srinivasan, V.,Kriete A.,Sacan A., Jazwinski S.M., Comparing the yeast retrograde response and NFkappaB stress responses: implications for aging. Aging Cell 2010, 9, 933-941.

40. Torelli, N.Q.,Ferreira-Junior J.R.,Kowaltowski A.J., da Cunha F.M.,RTG1 - and RTG2 -dependent retrograde signaling controls mitochondrial activity and stress resistance in Saccharomyces cerevisiae. Free Radic Biol Med 2015, 81, 30-37.

41. Komeili, A.,Wedaman K.P.,O'Shea E.K., Powers T., Mechanism of metabolic control. Target of rapamycin signaling links nitrogen quality to the activity of the Rtg1 and Rtg3 transcription factors. J Cell Biol 2000, 151, 863-878.

42. Liu, Z.C.,Sekito T.,Spirek M.,Thornton J.et al., Retrograde signaling is regulated by the dynamic interaction between Rtg2p and Mks1p. Molecular Cell 2003, 12, 401-411.

43. Liu, Z.,Spirek M.,Thornton J., Butow R.A., A novel degron-mediated degradation of the RTG pathway regulator, Mks1p, by SCFGrr1. Mol Biol Cell 2005, 16, 4893-4904.

44. Liu, Z., Butow R.A., Mitochondrial retrograde signaling. Annu Rev Genet 2006, 40, 159-185.

45. Ruiz-Roig, C.,Noriega N.,Duch A.,Posas F.et al., The Hog1SAPK controls the Rtg1 / Rtg3 transcriptional complex activity by multiple regulatory mechanisms. Mol Biol Cell 2012, 23, 4286-4296.

46. Laera, L.,Guaragnella N.,Zdralevic M.,Marzulli D.et al., The transcription factors ADR1 or CAT8 are required for RTG pathway activation and evasion from yeast acetic acid-induced programmed cell death in raffinose. Microb Cell 2016, 3, 621-631.

47. Nasution, O.,Lee Y.M.,Kim E.,Lee Y.et al., Overexpression of OLE1 enhances stress tolerance and constitutively activates the MAPK HOG pathway in Saccharomyces cerevisiae . Biotechnol Bioeng 2017, $114,620-631$.

48. Dong, S.J.,Yi C.F., Li H., Changes of Saccharomyces cerevisiae cell membrane components and promotion to ethanol tolerance during the bioethanol fermentation. Int J Biochem Cell Biol 2015, 69, 196-203.

49. Stukey, J.E.,Mcdonough V.M., Martin C.E., Isolation and Characterization of Ole1 , a Gene Affecting Fatty-Acid Desaturation from Saccharomyces-Cerevisiae. Journal of Biological Chemistry 1989, 264, 1653716544.

50. Pascual-Ahuir, A.,Manzanares-Estreder S., Proft M., Pro- and Antioxidant Functions of the PeroxisomeMitochondria Connection and Its Impact on Aging and Disease. Oxid Med Cell Longev 2017, 2017, 9860841. 
51. Zeeshan, H.M.,Lee G.H.,Kim H.R., Chae H.J., Endoplasmic Reticulum Stress and Associated ROS. Int J Mol Sci 2016, 17, 327.

52. Zhao, Y.Y.,Cao C.L.,Liu Y.L.,Wang J.et al., Genetic analysis of oxidative and endoplasmic reticulum stress responses induced by cobalt toxicity in budding yeast. Biochim Biophys Acta Gen Subj 2020, 1864, 129516.

53. Ramos, A.,Dos Santos M.M.,de Macedo G.T.,Wildner G.et al., Methyl and Ethylmercury elicit oxidative stress and unbalance the antioxidant system in Saccharomyces cerevisiae. Chem Biol Interact2020, 315, 108867.

54. Liu, Z.L., Ma M., Pathway-based signature transcriptional profiles as tolerance phenotypes for the adapted industrial yeastSaccharomyces cerevisiae resistant to furfural and HMF.Appl Microbiol Biotechnol 2020, 104, 3473-3492.

55. Simaan, H.,Lev S., Horwitz B.A., Oxidant-Sensing Pathways in the Responses of Fungal Pathogens to Chemical Stress Signals. Front Microbiol 2019, 10, 567.

56. Malina, C.,Larsson C., Nielsen J., Yeast mitochondria: an overview of mitochondrial biology and the potential of mitochondrial systems biology. FEMS Yeast Res 2018, 18.

57. Gruhlke, M.C.H.,Schlembach I.,Leontiev R.,Uebachs A.et al., Yap1p, the central regulator of the $S$. cerevisiae oxidative stress response, is activated by allicin, a natural oxidant and defence substance of garlic. Free Radic Biol Med 2017, 108, 793-802.

58. Wu, G.,Xu Z., Jonsson L.J., Profiling of Saccharomyces cerevisiae transcription factors for engineering the resistance of yeast to lignocellulose-derived inhibitors in biomass conversion.Microb Cell Fact 2017, 16, 199.

59. Qu, Z.,Zhang L.,Zhu S.,Yuan W.et al., Overexpression of the transcription factor HAC1 improves nerolidol production in engineered yeast. Enzyme Microb Technol 2020, 134, 109485.

60. Tran, D.M.,Ishiwata-Kimata Y.,Mai T.C.,Kubo M.et al., The unfolded protein response alongside the diauxic shift of yeast cells and its involvement in mitochondria enlargement. Sci Rep 2019, 9, 12780.

61. Mat Nanyan, N.S.B., Takagi H., Proline Homeostasis inSaccharomyces cerevisiae : How Does the StressResponsive Transcription Factor Msn2 Play a Role? Front Genet 2020, 11, 438.

62. Li, L.,Kaplan J., Ward D.M., The glucose sensor Snf1 and the transcription factors Msn2 and Msn4 regulate transcription of the vacuolar iron importer gene $\mathrm{CCC} 1$ and iron resistance in yeast. $J$ Biol Chem 2017, 292, 15577-15586.

63. Mira, N.P.,Becker J.D., Sa-Correia I., Genomic expression program involving the Haa1p-regulon in Saccharomyces cerevisiae response to acetic acid. OMICS 2010, 14, 587-601.

64. Sakihama, Y.,Hasunuma T., Kondo A., Improved ethanol production from xylose in the presence of acetic acid by the overexpression of the HAA1 gene in Saccharomyces cerevisiae. J Biosci Bioeng 2015, 119, 297-302.

65. Cunha, J.T.,Costa C.E.,Ferraz L.,Romani A.et al., HAA1 and PRS3 overexpression boosts yeast tolerance towards acetic acid improving xylose or glucose consumption: unravelling the underlying mechanisms. Appl Microbiol Biotechnol 2018, 102, 4589-4600.

66. Chen, Y.,Stabryla L., Wei N., Improved Acetic Acid Resistance inSaccharomyces cerevisiae by Overexpression of the WHI2Gene Identified through Inverse Metabolic Engineering. Appl Environ Microbiol 2016, $82,2156-2166$.

67. Kim, J.E.,Jang I.S.,Son S.H.,Ko Y.J.et al., Tailoring theSaccharomyces cerevisiae endoplasmic reticulum for functional assembly of terpene synthesis pathway. Metab Eng 2019, 56, 50-59. 
68. Qin, L.,Dong S.,Yu J.,Ning X.et al., Stress-driven dynamic regulation of multiple tolerance genes improves robustness and productive capacity of Saccharomyces cerevisiae in industrial lignocellulose fermentation. Metab Eng 2020.

69. Wei, S.,Liu Y.,Wu M.,Ma T.et al., Disruption of the transcription factors Thi2p and Nrm1p alleviates the post-glucose effect on xylose utilization in Saccharomyces cerevisiae. Biotechnol Biofuels2018, 11, 112.

70. Wei, S.,Bai P.,Liu Y.,Yang M.et al., A Thi2p Regulatory Network Controls the Post-glucose Effect of Xylose Utilization inSaccharomyces cerevisiae . Front Microbiol 2019, 10, 1649.

71. Fang, T.,Yan H.,Li G.,Chen W.et al., Chromatin remodeling complexes are involvesd in the regulation of ethanol production during static fermentation in budding yeast. Genomics 2020, 112, 1674-1679.

72. Peterson, C.L.,Zhao Y., Chait B.T., Subunits of the yeast SWI/SNF complex are members of the actin-related protein (ARP) family. J Biol Chem 1998, 273, 23641-23644.

73. Peterson, C. L, Herskowitz, I., Characterization of the yeast SWI1 , SWI2 , and SWI3 genes, which encode a global activator of transcription. Cell 1992, 68.

74. Antonets, K.S.,Kliver S.F.,Polev D.E.,Shuvalova A.R.et al., Distinct Mechanisms of Phenotypic Effects of Inactivation and Prionization of Swi1 Protein in Saccharomyces cerevisiae . Biochemistry (Mosc) 2017, $82,1147-1157$.

75. Zhang, Y.,Anderson S.J.,French S.L.,Sikes M.L.et al., The SWI/SNF chromatin remodeling complex influences transcription by RNA polymerase I in Saccharomyces cerevisiae . PLoS One 2013, 8, e56793.

76. Dastidar, R.G.,Hooda J.,Shah A.,Cao T.M.et al., The nuclear localization of SWI/SNF proteins is subjected to oxygen regulation. Cell Biosci 2012, 2, 30.

77. Li, P.,Fu X.,Zhang L.,Zhang Z.et al., The transcription factorsHsf1 and Msn2 of thermotolerant Kluyveromyces marxianus promote cell growth and ethanol fermentation of Saccharomyces cerevisiae at high temperatures. Biotechnol Biofuels 2017, 10, 289.

78. Veri, A.O.,Robbins N., Cowen L.E., Regulation of the heat shock transcription factor Hsf1 in fungi: implications for temperature-dependent virulence traits. FEMS Yeast Res 2018, 18.

79. Michael, D.G.,Maier E.J.,Brown H.,Gish S.R.et al., Model-based transcriptome engineering promotes a fermentative transcriptional state in yeast. Proc Natl Acad Sci U S A 2016, 113, E7428-E7437.

80. Liu, H.,Yan M.,Lai C.,Xu L.et al., gTME for improved xylose fermentation of Saccharomyces cerevisiae . Appl Biochem Biotechnol 2010, 160, 574-582.

81. Srivastava, R.,Rai K.,Pandey B.,Singh S.et al., Spt-Ada-Gcn5-Acetyltransferase (SAGA) Complex in Plants: Genome Wide Identification, Evolutionary Conservation and Functional Determination. PLOS ONE 2015, 10, e0134709-.

82. Canzonetta, C.,Leo M.,Guarino S.R.,Montanari A.et al., SAGA complex and Gcn5 are necessary for respiration in budding yeast.Biochim Biophys Acta 2016, 1863, 3160-3168.

83. Warfield, L.,Ramachandran S.,Baptista T.,Devys D.et al., Transcription of Nearly All Yeast RNA Polymerase II-Transcribed Genes Is Dependent on Transcription Factor TFIID. Mol Cell 2017, 68, 118-129 e115.

84. Ansari, S.A.,Ganapathi M.,Benschop J.J.,Holstege F.C.et al., Distinct role of Mediator tail module in regulation of SAGA-dependent, TATA-containing genes in yeast. EMBO J 2012, 31, 44-57.

85. Xue, T.,Chen D.,Su Q.Q.,Yuan X.et al., Improved ethanol tolerance and production of Saccharomyces cerevisiae by global transcription machinery engineering via directed evolution of the SPT8 gene. Food Biotechnol 2019, 33, 155-173. 
86. Zhu, L.,Gao S.,Zhang H.,Huang H.et al., Improvement of Lead Tolerance of Saccharomyces cerevisiae by Random Mutagenesis of Transcription Regulator SPT3 . Appl Biochem Biotechnol2018, 184, 155-167.

87. Phithakrotchanakoon, C.,Puseenam A.,Wongwisansri S.,Eurwilaichitr L.et al., CRISPR-Cas9 enabled targeted mutagenesis in the thermotolerant methylotrophic yeast Ogataea thermomethanolica. FEMS Microbiol Lett 2018, 365.

88. Urnov, F.D., Rebar E.J., Designed transcription factors as tools for therapeutics and functional genomics. Biochemical Pharmacology2002, 64, 919-923.

89. Heiderscheit, E.A.,Eguchi A.,Spurgat M.C., Ansari A.Z., Reprogramming cell fate with artificial transcription factors.FEBS Lett 2018, 592, 888-900.

90. Chattopadhyay, A.,Purohit J.,Tiwari K.K., Deshmukh R., Targeting transcription factors for plant disease resistance: shifting paradigm. Current science 2019, 117, 1598-1607.

91. Liu, W., Stewart C.N., Jr., Plant synthetic promoters and transcription factors. Curr Opin Biotechnol 2016, 37, 36-44.

92. Purcell, O.,Peccoud J., Lu T.K., Rule-based design of synthetic transcription factors in eukaryotes. ACS Synth Biol 2014, 3, 737-744.

93. Klug, A., The discovery of zinc fingers and their development for practical applications in gene regulation and genome manipulation. $Q$ Rev Biophys 2010, 43, 1-21.

94. Lee, J.Y.,Sung B.H.,Yu B.J.,Lee J.H.et al., Phenotypic engineering by reprogramming gene transcription using novel artificial transcription factors in Escherichia coli. Nucleic Acids Res 2008, 36, e102.

95. Lee, J.Y.,Yang K.S.,Jang S.A.,Sung B.H.et al., Engineering butanol-tolerance in escherichia coli with artificial transcription factor libraries. Biotechnol Bioeng 2011, 108, 742-749.

96. Park, K.S.,Lee D.K.,Lee H.,Lee Y.et al., Phenotypic alteration of eukaryotic cells using randomized libraries of artificial transcription factors. Nat Biotechnol 2003, 21, 1208-1214.

97. Ma, C.,Wei X.,Sun C.,Zhang F.et al., Improvement of acetic acid tolerance of Saccharomyces cerevisiae using a zinc-finger-based artificial transcription factor and identification of novel genes involved in acetic acid tolerance. Appl Microbiol Biotechnol2015, 99, 2441-2449.

98. Rantasalo, A.,Kuivanen J.,Penttila M.,Jantti J.et al., Synthetic Toolkit for Complex Genetic Circuit Engineering in Saccharomyces cerevisiae. ACS Synth Biol 2018, 7, 1573-1587.

99. Naseri, G.,Balazadeh S.,Machens F.,Kamranfar I.et al., Plant-Derived Transcription Factors for Orthologous Regulation of Gene Expression in the Yeast Saccharomyces cerevisiae . ACS Synth Biol 2017, 6, $1742-1756$.

100. Naseri, G.,Behrend J.,Rieper L., Mueller-Roeber B., COMPASS for rapid combinatorial optimization of biochemical pathways based on artificial transcription factors. Nat Commun 2019, 10, 2615.

101. Machens, F.,Balazadeh S.,Mueller-Roeber B., Messerschmidt K., Synthetic Promoters and Transcription Factors for Heterologous Protein Expression in Saccharomyces cerevisiae. Front Bioeng Biotechnol 2017, 5, 63 .

102. Rantasalo, A.,Landowski C.P.,Kuivanen J.,Korppoo A.et al., A universal gene expression system for fungi. Nucleic Acids Res2018, 46, e111.

103. Nguyen, N.H.,Kim J.-R., Park S., Development of Biosensor for 3-Hydroxypropionic Acid. Biotechnology and Bioprocess Engineering2019, 24, 109-118.

104. Durai, L.,Vijayalakshmi R., Karunagaran D., A novel reporter system for cyclic AMP mediated gene expression in mammalian cells based on synthetic transgene expression system. Eur J Pharmacol 2019, 855, 
$56-64$.

105. Li, J.W.,Zhang X.Y.,Wu H., Bai Y.P., Transcription Factor Engineering for High-Throughput Strain Evolution and Organic Acid Bioproduction: A Review. Front Bioeng Biotechnol 2020, 8, 98.

106. Myers, K.S.,Riley N.M.,MacGilvray M.E.,Sato T.K.et al., Rewired cellular signaling coordinates sugar and hypoxic responses for anaerobic xylose fermentation in yeast. PLoS Genet 2019, 15, e1008037.

107. Yang, T.H., Transcription factor regulatory modules provide the molecular mechanisms for functional redundancy observed among transcription factors in yeast. BMC Bioinformatics 2019, 20, 630.

108. Thorwall, S.,Schwartz C.,Chartron J.W., Wheeldon I., Stress-tolerant non-conventional microbes enable next-generation chemical biosynthesis. Nat Chem Biol 2020, 16, 113-121.

109. Xu, K.,Qin L.,Bai W.X.,Wang X.Y.et al., Multilevel Defense System (MDS) Relieves Multiple Stresses for Economically Boosting Ethanol Production of Industrial Saccharomyces cerevisiae. Acs Energy Lett 2020, 5, 572-582.

110. Kuki, Y.,Ohno R.,Yoshida K., Takumi S., Heterologous expression of wheat WRKY transcription factor genes transcriptionally activated in hybrid necrosis strains alters abiotic and biotic stress tolerance in transgenic Arabidopsis. Plant Physiol Biochem 2020, 150, 71-79.

111. Nghia, D.H.T.,Chuong N.N.,Hoang X.L.T.,Nguyen N.C.et al., Heterologous Expression of a Soybean Gene RR34 Conferred Improved Drought Resistance of Transgenic Arabidopsis. Plants (Basel)2020, 9 (4), 494.

112. Wang, L.,Liu W., Wang Y., Heterologous expression of Chinese wild grapevine VqERFs in Arabidopsis thaliana enhance resistance to Pseudomonas syringae pv. tomato DC3000 and to Botrytis cinerea.Plant Sci 2020, 293, 110421.

113. Yao, L.,Hao X.,Cao H.,Ding C.et al., ABA-dependent bZIP transcription factor, CsbZIP18 , from Camellia sinensis negatively regulates freezing tolerance in Arabidopsis. Plant Cell Rep 2020, 39, 553-565.

114. Du, X.,He F.,Zhu B.,Ren M.et al., NAC transcription factors from Aegilops markgrafii reduce cadmium concentration in transgenic wheat.Plant and Soil 2020, 449, 39-50.

115. Wan, F.,Pan Y.,Li J.,Chen X.et al., Heterologous expression of Arabidopsis C-repeat binding factor 3 (AtCBF3 ) and cold-regulated 15A (AtCOR15A) enhanced chilling tolerance in transgenic eggplant (Solanum melongena L.). Plant Cell Rep 2014, 33, 1951-1961.

116. Zhao, P.,Hou S.,Guo X.,Jia J.et al., A MYB-related transcription factor from sheepgrass, LcMYB2 , promotes seed germination and root growth under drought stress. BMC Plant Biol 2019, 19, 564.

Table 1. Heterogeneous TFs applications in Plants

\begin{tabular}{|c|c|c|c|c|}
\hline $\begin{array}{l}\text { Transcription } \\
\text { factor }\end{array}$ & Source & $\begin{array}{l}\text { Engineered } \\
\text { method }\end{array}$ & Values & References \\
\hline $\begin{array}{l}\text { TaWRKY49 } \\
\text { TaWRKY92 } \\
\text { TaWRKY112 } \\
\text { TaWRKY142 }\end{array}$ & Wheat & $\begin{array}{l}\text { Heterologous } \\
\text { expression }\end{array}$ & $\begin{array}{l}\text { Increased salinity } \\
\text { and osmotic stress } \\
\text { tolerance and } \\
\text { promoted } \\
\text { STZ/Zat10, AtPR1 } \\
\text { expression }\end{array}$ & {$[110]$} \\
\hline RR34 & Glycinemax & & $\begin{array}{l}\text { Improve drought } \\
\text { resistance of } \\
\text { transgenic } \\
\text { arabidopsis }\end{array}$ & {$[111]$} \\
\hline
\end{tabular}




\begin{tabular}{|c|c|c|c|c|}
\hline $\begin{array}{l}\text { Transcription } \\
\text { factor }\end{array}$ & Source & $\begin{array}{l}\text { Engineered } \\
\text { method }\end{array}$ & Values & References \\
\hline $\begin{array}{l}\text { VqERF112 } \\
\text { VqERF114 } \\
\text { VqERF072 }\end{array}$ & $\begin{array}{l}\text { Wild Vitis } \\
\text { quinquangularis }\end{array}$ & & $\begin{array}{l}\text { Improve the } \\
\text { resistance of } \\
\text { Arabidopsis to } \\
\text { pathogenic bacteria }\end{array}$ & {$[112]$} \\
\hline CsbZIP18 & Camellia sinensis & & $\begin{array}{l}\text { Negatively } \\
\text { regulates freezing } \\
\text { tolerance in } \\
\text { Arabidopsis }\end{array}$ & {$[113]$} \\
\hline$N A C 2 / 3$ & $\begin{array}{l}\text { Aegilops } \\
\text { markgrafii }\end{array}$ & & $\begin{array}{l}\text { Reduce Cd } \\
\text { concentration in } \\
\text { the root, shoot } \\
\text { and grains }\end{array}$ & {$[114]$} \\
\hline AtCBF3 & Arabidopsis & & $\begin{array}{l}\text { Improve cold } \\
\text { tolerance in } \\
\text { transgenic } \\
\text { eggplant }\end{array}$ & {$[115]$} \\
\hline$L c M Y B 2$ & Sheepgrass & & $\begin{array}{l}\text { Improve the } \\
\text { drought and } \\
\text { osmotic tolerance } \\
\text { of plants }\end{array}$ & {$[116]$} \\
\hline
\end{tabular}

\section{Figure legends}

Figure 1. The molecular mechanisms of yeast underlying high temperature response and thermotolerance

(1) Under normal circumstances, Hsf1p is inhibited by binding to Hsp70 which the association is weak. In response to high temperature, Hsflp is released by binding of Hsp70p with misfolded protein, which then phosphorylates and imported into the nucleus recruiting mediators (Head, Middle, Tail, and Kinase) to increase the amount of RNA Pol II occupied and reconstructing the chromatin distribution in the promoters. The mediator is a conserved transcriptional coactivation complex. Its component subunits of Med2p, Med3p, and Med15p can recruit on regulatory regions of prompter, which enables smooth expression of target genes involving heat shock protein (HSPs), protein homeostasis and trehalose synthesis. (2) The number of new ribosomal proteins (RPs) and rRNAs do not match, resulting in the orphan r-proteins aggregate. Hsf1p is activated by them, which then stimulate the expression of protein homeostasis genes. On the other hand, Ifh1p also activated by orphan r-proteins, which in turn inhibits the synthesis of ribosomal protein genes. (3) Ras-PKA pathway influence the activation of Msn2/3p by phosphorylate. Stress leads Msn2/3p dephosphorylated, and then enters the nucleus and activate the downstream regulated genes expression which have stress response elements (STREs)

Figure 2. Deleterious effects of ethanol and acetic acid on yeast cells and cellular adaptive response to stress

(1) Fps1p is the aquaglyceroporin channel which transports the acetic acid from extracellular to cytoplasm, and it can interact with regulatory proteins $\operatorname{Rgc1p} / \operatorname{Rgc} 2 \mathrm{p}$. To cope with acetic acid stress, Fps1p is closed in response to increases in cellular acetic acid concentration. And other ATPase-dependent transporters (Pdr10, Pdr12, Pma1) seem to play an important role in maintaining intracellular concentration of $\mathrm{CH}_{3} \mathrm{COOH}^{-} / \mathrm{H}^{+}$homeostasis. (2) Hyperphosphorylated Mks1p binds to Bmh1/2p, inhibiting the Rtg3p and Rtg1p complexes translocate to nucleus and inhibits the retrograde signals transmission in normal conditions. When the strains exposure to acetic acid, Mitochondrial dysfunction leads to a decrease in ATP content. And, Mks1p and Rtg2p are combined to inhibit the Mks1-Bmh1/2p complex function of inhibiting 
the RTG pathway, resulting in the activated Rtg3p-Rtg1p complex entering the cell nucleus to regulate RTG-dependent nuclear genes expression such as IDH1, IDH12, DLD3, PYC1. (3) HAA1 is sensitive to intracellular acetic acid. After too much acetic acid dissociated in the cell to form acetate, it will be activated by dephosphorylation, thus avoiding anionic toxicity. (4) To deal with cell membrane destruction caused by ethanol, Ssk1p which is Cytoplasmic phosphorelay intermediate regulator is activated by overexpressing Ole1p, then sequentially activates Ssk2/22p and Pbs2p. Finally, HOG pathway is stimulated by Pbs2p in response to ethanol stress.

Figure 3. Functional reaction to oxidant and Toxic molecule stress signals

(1) The activation of Yap1p is dependent or not on ROS caused by oxidizing chemicals, it transits from cytoplasm to nucleus to stimulate the expression of oxidative stress responsive gene such as GSH, GSN, SOD2 and GLR1. And, Hyr1p or Ybp1p-Gpx3p complex can inhibit YAP1 output from nuclear. (2) The unfolded protein response (UPR) signaling pathway mainly contains two regulators, i.e., the Ire1p and Hac1p. The original Hac1p mRNA contains introns which will inhibit its transcription activity. Once Ire1p releases from upon ER stress, Hac1p introns is cut to stimulate the expression of UPR genes expression. (3) The glutathione (GSH) and thioredoxin (TRX) system be respectively regard as representative of the nonenzymatic antioxidant system and enzyme antioxidant system. The sulfydryl of GSH has reducing activity interact with oxidizing chemicals to form oxidized glutathione (GSSG). Using glutathione reductase and NADPH to restore GSSG to GSH. And, the thioredoxin system includes thioredoxin reductase, thioredoxin and thioredoxin peroxidase. Thioredoxin (TPX) is a class of thiol-rich proteins. It can be used as a reducing substrate to maintain intracellular redox together with thioredoxin reductase. (3) The glutathione (GSH) and thioredoxin (TRX) system be respectively regard as representative of the non-enzymatic antioxidant system and enzyme antioxidant system. The sulfydryl of GSH has reducing activity interact with oxidizing chemicals to form oxidized glutathione (GSSG). Using glutathione reductase and NADPH to restore GSSG to GSH. And, the thioredoxin system includes thioredoxin reductase, thioredoxin and thioredoxin peroxidase. Thioredoxin (TPX) is a class of thiol-rich proteins. It can be used as a reducing substrate to maintain intracellular redox together with thioredoxin reductase.

Figure 4. Transcription Factor Engineering used to improve strains industrial value and create new tools

(A) The conventional gene modifying methods including overexpression and knockout can be brought into reconstructing metabolic networks, especially in stress defense system. (B) gTME and heterologous expression based on multi-TF structures provides a possibility for promoting self-activity and enhancing gene transcription level that contribute to the tolerance and productivity. (C) ATF together with their cognate binding sites $(\mathrm{CBS})$ and synthetic promoter $\left(\mathrm{P}_{\mathrm{Syn}}\right)$ construct an orthorhombic gene expression system to regulate the genes expression. (D) TF-based biosensors consist of effector domain (ED) for sensing small molecules and DNA-binding domain (DBD) combined with DNA, which provides more opportunities to quantitatively evaluating substance content. 

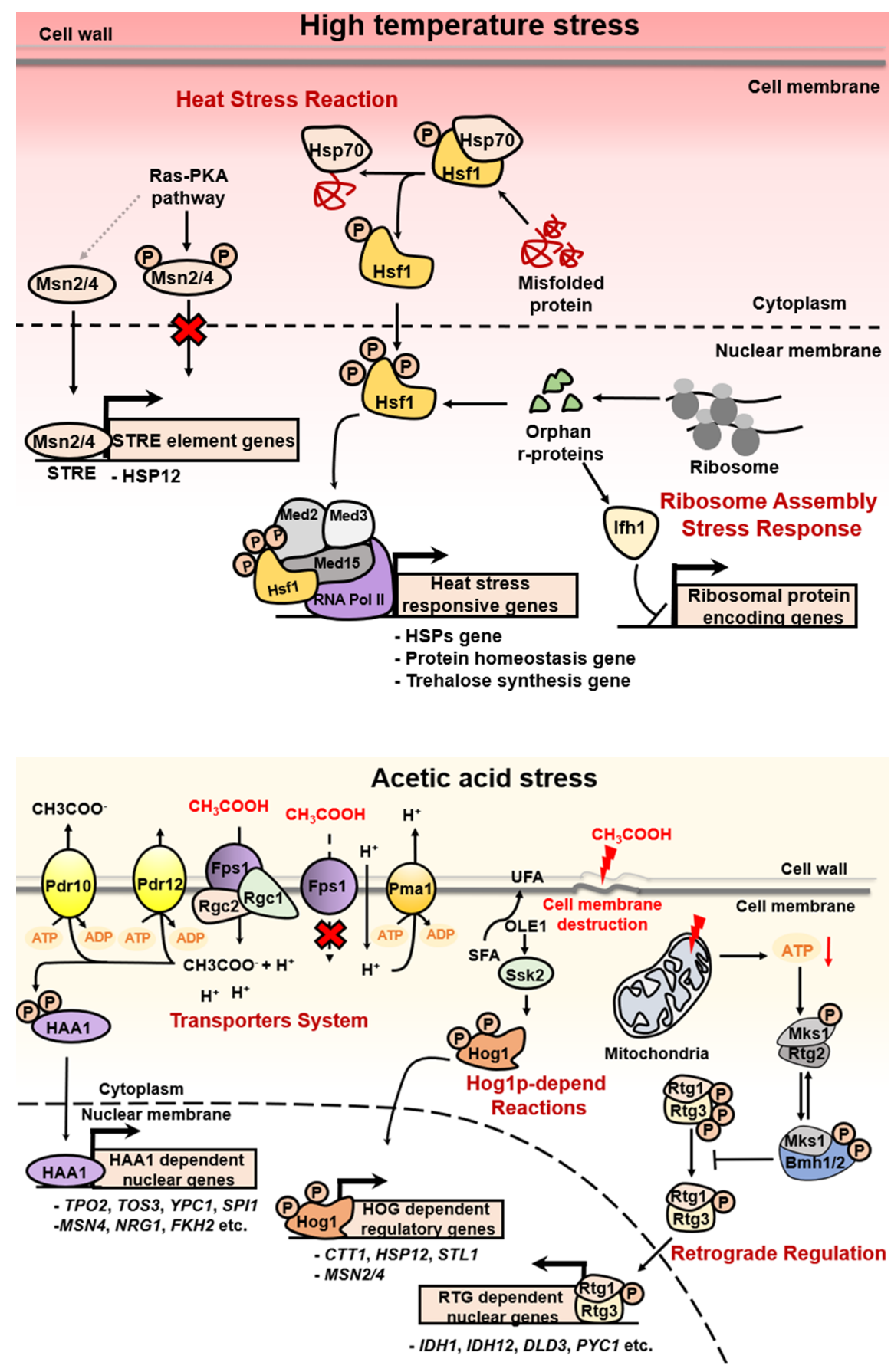


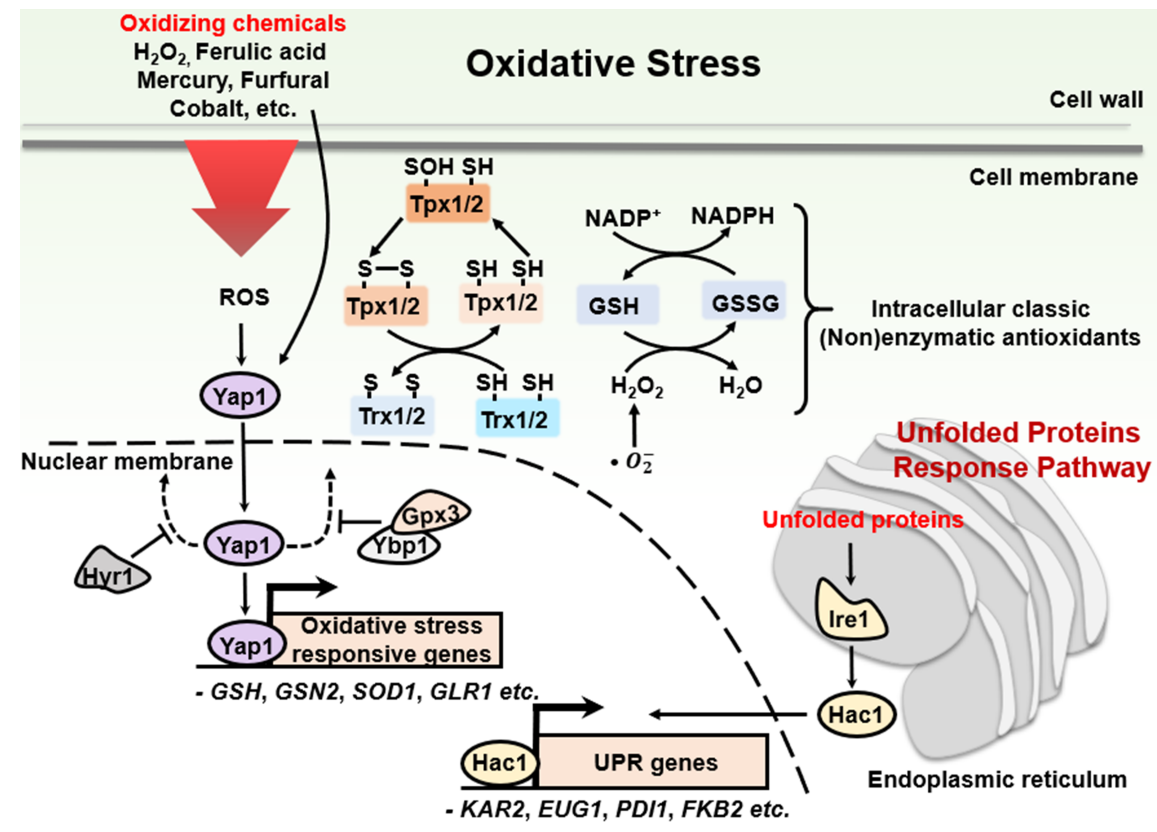

A Conventional Transcription Factor Engineering
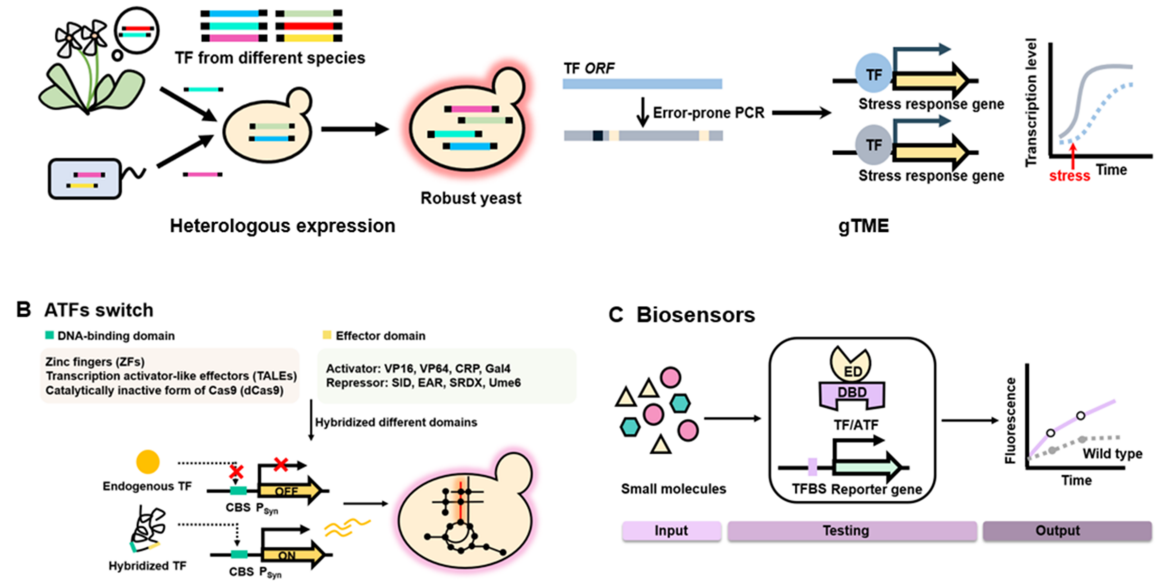